\title{
CLASSIFICATION OF TEXTURES SEEN FROM DIFFERENT DISTANCES AND UNDER VARYING ILLUMINATION DIRECTION
}

\author{
Xavier Lladó, Joan Martí \\ Computer Vision and Robotics Group, \\ Institute of Informatics and Applications, \\ University of Girona, 17071, Girona, Spain \\ \{llado, joanm\}@eia.udg.es
}

\begin{abstract}
Changes in the angle of illumination incident upon a $3 \mathrm{D}$ surface texture can significantly alter its appearance, implying variations in the image texture. These texture variations produce displacements of class members in the feature space, increasing the failure rates of texture classifiers. To avoid this problem, a model-based texture recognition system which classifies textures seen from different distances and under different illumination directions is presented in this paper. The system works on the basis of a surface model obtained by means of 4-source Colour Photometric Stereo, used to generate $2 \mathrm{D}$ image textures under different illumination directions. The recognition system combines co-ocurrence matrices for feature extraction with a Nearest Neighbour classifier. Moreover, the recognition allows one to guess the approximate direction of the illumination used to capture the test image.
\end{abstract}

\section{INTRODUCTION}

The main motivation of this paper is the necessity to find methods to reduce texture classification errors caused by changes in illuminant direction. The 2-dimensional texture in the image, the image texture, is produced by variation in both surface reflectance and surface relief. While the reflectance properties are intrinsic to the surface, the surface relief produces a pattern of shadings that depends strongly on the direction of the illumination [1]. Thus, the image texture created by a 3D surface texture changes drastically with the imaging geometry as illustrated in fig. 1 .

These changes in the illumination can introduce critical misclassification rates. if the illumination direction is altered between training and classification. Most of the classification approaches proposed in the literature do not take into account the effect of illumination on the imaged scene,

This work was supported by UdG grant BRO0/03 and by EPSRC grant GR/M73361. M Petrou is on leave of absence from the University of Surrey, UK.

\author{
Maria Petrou \\ Informatics and Telematic Institute, \\ EKETA, PO Box 361, Thermi, \\ Thessaloniki 57001, Greece \\ petrou@iti.gr
}

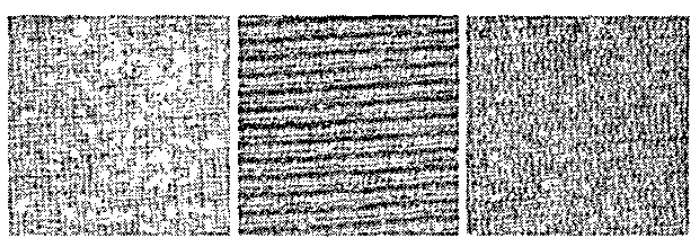

Fig. 1. Three images of the same surface texture sample captured using different illuminant tilt angles.

thus tacitly assuming that the illumination direction is constant. Very few works have been published on this topic. Leung and Malik [2] developed a texture classification scheme that identifies 3D "textons" in the Columbia-Utrecht database for the purpose of illumination and viewpoint invariant classification. Recently, Chantler et al. [3] presented a formal theory which demonstrates that changes in the tilt of the illumination direction make texture features to follow superelliptical trajectories in multi-dimensional feature spaces. Based on their work, Penirschke et al. [4] developed an illuminant rotation invariant classification scheme. This work is based on the use of photometric stereo for the detection of surface relief, and the use of Gabor features. The production of features by filtering, however, requires the use of all points of the surface/image, the surface gradient of some of which may have been wrongly calculated by the photometric stereo technique. In this paper, we also rely on the use of photometric stereo, but we use features constructed from cooccurrence matrices which can be computed by using only the points for which the photometric stereo technique produces reliable results. In addition, the model-based texture recognition system we present is able to cope with changes in the direction of illumination (changes in the tilt angle ${ }^{1}$ ),

\footnotetext{
${ }^{1}$ We define our camera axis parallel to the z-axis. Therefore, the illuminant tilt is the angle the illuminant vector makes with the $x$-axis when it is projected onto the $x, y$ plane, ie the image plane. The illuminant slant is the angle that the illuminant vector makes with the camera axis.
} 
as well as changes in the distance of the camera ${ }^{2}$.

The surface model obtained is derived by using Colour Photometric Stereo (CPS) [5]. From this photometric information and by using an image distance prediction method described in [6], image textures of the surfaces at a longer distance from the camera and for various directions of illumination are generated. These image textures are used as models in the classification process. Moreover, this modelbased recognition allows one to guess the approximate direction of the illumination under which the test image was captured.

\section{RECOGNITION SYSTEM}

The whole procedure of the texture recognition system is illustrated in fig. 2 . The procedure is divided in two main phases: virtual image generation and recognition.

\subsection{Virtual database creation}

In the virtual database generation, 4-source CPS described in [5] is used to compute the detailed shape and colour of a rough surface when seen by a camera at the zenith of the surface. We assume that all the information concerning the surface texture constructed from the CPS is available in a database. From this photometric information a new set of texture images imaged under different directions of illumination is generated. This database (we shall refer to as "virtual" database) is used as a reference database for the purpose of classification.

Furthermore, we can extend the recognition of textures to the recognition of textures seen from a longer distance. A virtual database of images corresponding to the longer distance is generated using the image prediction method described in [6]. This method allows us to predict from the information extracted by CPS how an image of the surface would look like when seen from a longer distance and for any direction of illumination. Note that this is the image prediction process shown in the recognition scheme (see fig. 2). Omitting this process, the scheme illustrates the recognition of textures seen from the same distance.

\subsection{Recognition procedure}

The recognition procedure starts by extracting a representative feature vector for each texture image in the virtual database. The co-ocurrence matrices [7] are used to extract features as contrast, homogeneity and energy for 20 different values of a distance $d$ (distances between [1 . . 55] incremented by 3 steps are chosen in our experiments). The coocurrence matrices are implemented in an anisotropic way.

\footnotetext{
${ }^{2}$ A change in the camera distance only implies a change in the $z$ coor-
}

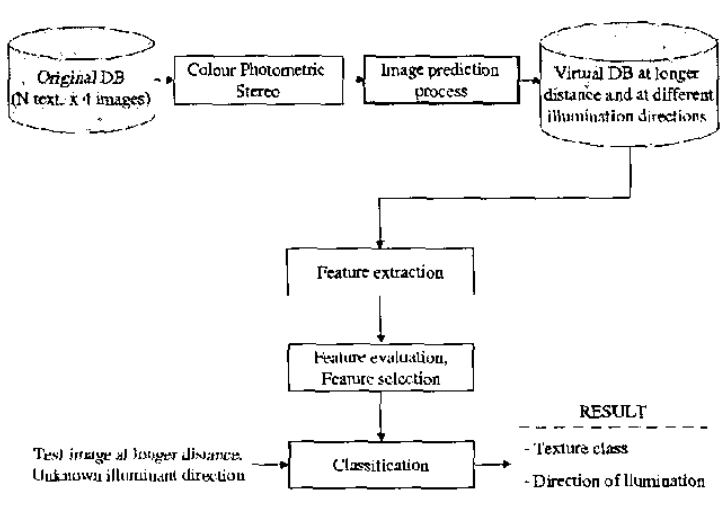

Fig. 2. Recognition scheme.

We analyse 4 different directions: $0^{\circ}, 45^{\circ}, 90^{\circ}, 135^{\circ}$, so we have in all 240 texture features ( 3 features $\times 4$ directions $\times 20$ distances). Among all the computed features, those which could discriminate between the different classes best are chosen. Due to the large number of features the Sequential Forward Selection (SFS) [8] algorithm is applied in order to find the best feature set. The feature evaluation is performed applying the Nearest Neighbour classifier over a set of training images. Therfore, the obtained classification rate gives us the measure of subset quality.

When the feature set is selected, the feature vectors for the unknown image textures (test images) are calculated and assigned to one of the classes of the virtual database, estimating also the direction of illumination.

\section{EXPERLMENTAL TRIALS}

The proposed recognition system was tested with 18 photometric sets (18 different surface textures) consisting of 4 images each. As well as of the 4 images used in the photometric sets, 12 different images for each surface were captured for testing. using 12 illuminant tilt angles between $0^{\circ}$ and $360^{\circ}$ incremented by steps of $30^{\circ}$. See the illumination set up in fig. 3.a. All surfaces were illuminated at a slant angle of $55^{\circ}$. These are the images we shall want to classify, using as reference images those generated by the photometric stereo sets. Moreover, another set of 12 test images for each surface were captured from a longer distance in order to evaluate the recognition at a different distance.

As it has been explained earlier, the main objectives are to improve the texture recognition and to evaluate the accuracy with which the system estimates the illuminant tilt angle of the light source. For the estimation of the tilt angle we used as reference in the virtual database four new illuminant tilt angles: $10^{\circ}, 100^{\circ}, 190^{\circ}$ and $280^{\circ}$ (see fig. 3.b). These 


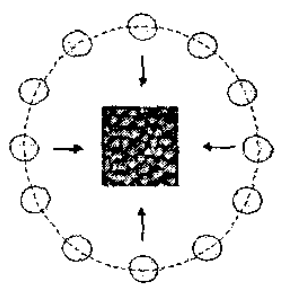

(a)

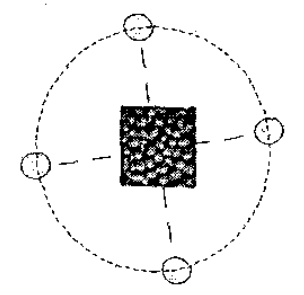

(b)
Fig. 3. Illumination setup. (a) Illuminant tilt angles used to capture the test images. (b) Illuminant tilt angIes used to create the virtual database. The unknown illuminant tilt angle of the test image has to be classified to the nearest one among these four angles used for the creation of the virtual database.

are the illuminant directions of the images generated from the photometric sets, and also the approximated tilt angles which the recognition gives us. Consequently, we classify test images imaged from 12 directions of illumination into the nearest texture class, and with one of the 4 illuminant tilt angles presented in the virtual database. Note that a virtual database of 72 image textures (18 surfaces $\times 4$ illuminant tilt angles) is created.

Using the data collected as described, we carry out two sets of experiments: in the first set we perform classification without image prediction, ie we use reference images and test images captured from the same distance. In this set of experiments we compare the results of our model-based approach with the results obtained in the "best" case, in which the models are real images captured with the same illumination angle as the test images, as well as with the "naive" case, in which just a single image of one illuminant direction is used to characterise each texture class. In the second set of experiments, we not only use test images captured with different illumination angle from those in the virtual database, but images which, in addition, have been captured from a longer distance than the distance used to capture the original photometric images.

\subsection{Classification without image prediction}

Due to the large size of the test images, we produced from each one of them different subimages of size $133 \times 133$, to be used for testing. During the feature selection process we used a training set of test images composed of $18 \times 12 \times 3=$ 648 test images (18 different surfaces, 12 different directions of illumination, 3 subimages). While for the classification purpose we used 9 subimages, obtaining a set of $18 \times 12 \times 9=1944$ test images.

Using our model-based approach we obtained a $97.48 \%$
Table 1. Texture and illuminant classification rates obtained for the best case, model-based and naive case.

\begin{tabular}{|c|c|c|c|c|}
\cline { 2 - 4 } & \multicolumn{2}{c|}{ Without image prediction } & \multicolumn{2}{c|}{ With image prediction } \\
\cline { 2 - 5 } & Texture & Illuminant & Texture & Illuminant \\
\hline Best case & $100 \%$ & $86.73 \%$ & $100 \%$ & $85.03 \%$ \\
\hline Model-based & $97.48 \%$ & $66.62 \%$ & $89.40 \%$ & $60.96 \%$ \\
\hline Naive case & $88.84 \%$ & & $81.22 \%$ & - \\
\hline
\end{tabular}

of texture classification, while we estimated correctly the illuminant tilt angle with an accuracy of $66.62 \%$. However, in the "best" case in which we used original images as references for the purpose of classification, the texture classification obtained was $100 \%$, while the illuminant classification was $86.73 \%$. For the "naive" case in which just one illuminant direction was used for training, the texture classification was $88.84 \%$ accurate. Note that using our model-based approach classification errors caused by changes in illuminant direction are reduced compared with the "naive" case.

\subsection{Classification with image prediction}

As we did previously, from each captured image we created 9 subimages to be used for testing. Thus, we had in all 1944 test images captured by the same camera but from a longer distance than those in the training set. After applying the feature selection algorithm and choosing the appropriate feature set, we applied the classifier to the unknown test images. Using our model-based approach we classified $89.40 \%$ of them into the correct texture class, while we estimated with an accuracy of $60.96 \%$ the illuminant tilt angle. As in the previous results, we arrived at $100 \%$ of texture classification using the "best" case, while the illuminant classification was $85.03 \%$. For the "naive" case the texture classification was $81.22 \%$.

\subsection{Discussion}

Table 1 summarises the obtained texture and illuminant classification rates using our model-based approach, the "best" case, and the "naive" case. Note that misclassification errors of the model-based approach without doing image distance prediction is presumed to be due to both inadequate image generation of CPS and limitations of the classifier itself, whereas the "best" case misclassification may be due solely to the latter. Note also that the results demonstrate that our model-based approach reduces the classification errors caused by changes in illuminant direction compared with the "naive" case. When the image distance prediction is used, all misclassification errors increased due to the error introduced by the image distance prediction method. 

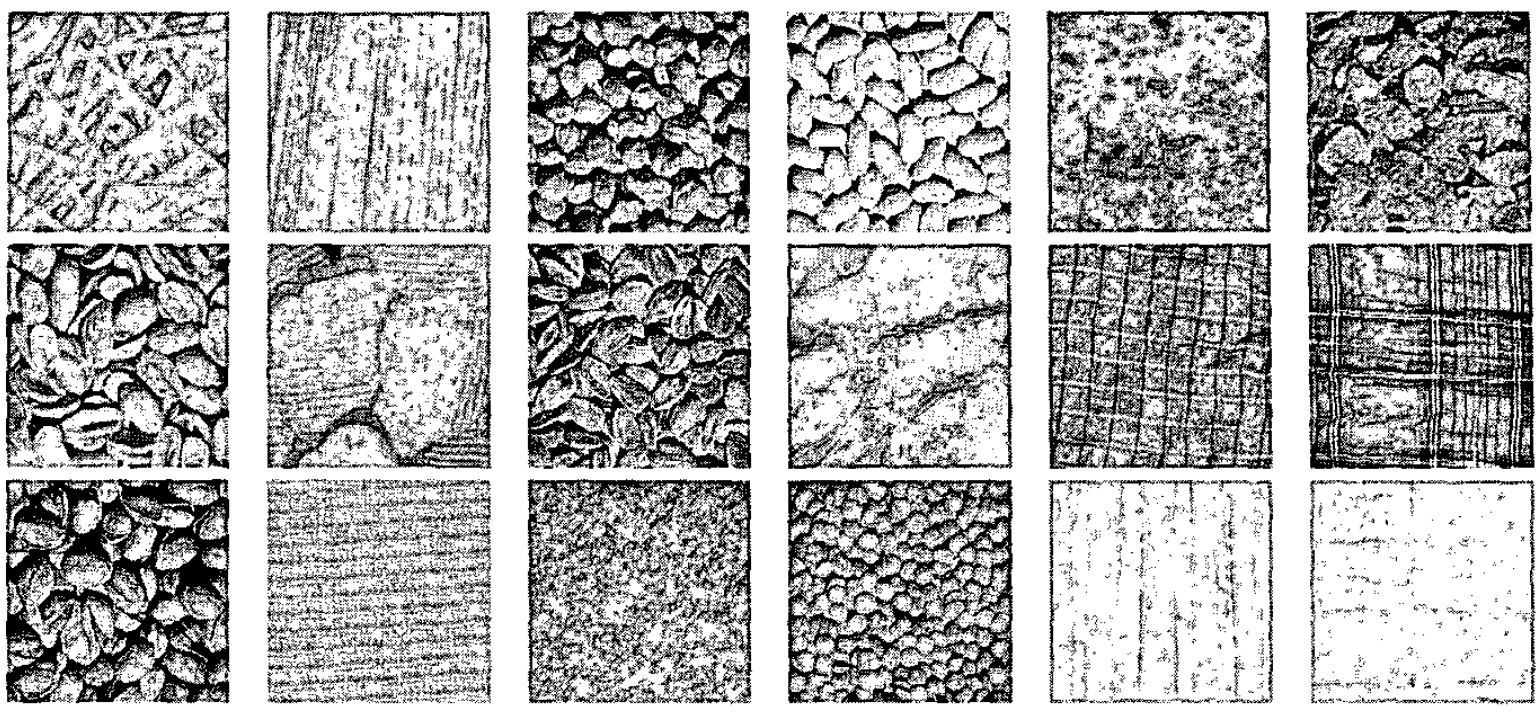

Fig. 4. One image of each of the eighteen sample textures.

However, good classification errors are achieved considering the errors produced by CPS, distance predicion method, and limitations of the classifier.

\section{SUMMARY AND CONCLUSIONS}

We presented a model-based texture recognition system able to classify textures when seen from different distances and under different ditections of illumination. The 4-source CPS has been used in order to obtain a surface model for each texture. Using this information and using an image prediction process, different image textures under various illuminant directions are generated. These images are used as a reference set for the classification purpose with the aim to reduce the classification errors caused by changes in illuminant direction. The results presented in this paper demonstrate the validity of the system, including its capacity to estimate approximately the direction of the light source.

\section{REFERENCES}

[1] M.J. Chantler, "Why illuminant direction is fundamental to texture analysis," IEE Proceedings in Vision, Image and Signal Processing, vol. 142, no. 4, pp. 199 206. August 1995.

[2] T. Leung and J. Malik, "Representing and recognising the visual appearance of materials using threedimensional textons.," International Journal of Computer Vision, vol. 43. no. 1, pp. 29-44. June 2001.
[3] M. Chantler, M. Schmidt, M. Petrou, and G. McGunnigle, "The effect of illuminant rotation on texture filters: Lissajous's ellipses," in 7th European Conference on Computer Vision, Copenhagen, Denmark, May 2002, vol. 3, pp. 289-304.

[4] A. Penirschke, M. Chantler, and M. Petrou, "Illuminant rotation invariant classification of $3 \mathrm{~d}$ surface textures using lissajous's ellipses," in 2nd international workshop on texture analysis and symthesis. Copenhagen, Denmark, June 2002, pp. 103-107.

[5] S. Barsky and M. Petrou, "The 4-source photometric stereo technique for 3-dimensional surfaces in the presence of highlights and shadows," IEEE Transactions on Pattern Analysis and Machine Intelligence, 2003.

[6] X. Llado and M. Petrou, "Predicting surface texture when seen from different distances," in 2nd International Workshop on Texture Analysis and Synthesis, Copenhagen, Denmark, June 2002, pp. 83-86.

[7] R.M. Haralick, K.S. Shanmugan, and I. Dunstein, "Textural features for image classification," IEEE Transactions on Systems, Man, and Cybertinetics, vol. 3, no. 6, pp. 610-621, 1973.

[8] J. Kittler, "Feature set search algorithms," in Pattern Recognition and Signal Processing. Sijthoff and Noordhoff, Alphen aan den Rijn. The Netherlands, 1978. pp. $41-60$. 